Pacific Journal of Mathematics

SOME RESULTS ON THE FLOQUET THEORY FOR 


\title{
SOME RESULTS ON THE FLOQUET THEORY FOR DISCONJUGATE DEFINITE HAMILTONIAN SYSTEMS
}

\author{
WILLIAM T. REID
}

\begin{abstract}
For a linear periodic Hamiltonian system that is definite, identically normal, and disconjugate on $(-\infty, \infty)$ there is established a basic relation between the Floquet representation of a fundamental matrix for this system and the distinguished solutions at $\pm \infty$ of the associated Riccati matrix differential equation.
\end{abstract}

1. Introduction. The present paper is a consequence of hearing R. S. Bucy present [4] of the References at the recent International Conference on Differential Equations at the University of California at Los Angeles, September 3-7, 1974, and the subsequent privilege of reading a preprint of the paper. The goal of the present paper is quite distinct from that of [4], however, as herein no attention is devoted to numerical processes, whereas Bucy is concerned with the development of a method which not only gives the characteristic exponents, but also allows for the numerical determination of the associated constant Hamiltonian system given by the Floquet theory. Further comparative comments on the hypotheses of the two papers are presented in $\S 5$.

Matrix notation is used throughout; in particular, one column matrices are called vectors. The $n \times n$ identity matrix is denoted by $E_{n}$, or merely by $E$ when there is no ambiguity, and 0 is used indiscriminately for the zero matrix of any dimensions. For a matrix $M$ the conjugate transpose is denoted by $M^{*}$. The notation $M \geqq N$, $\{M>N\}$, is used to signify that $M$ and $N$ are hermitian matrices of the same dimensions, and $M-N$ is a nonnegative, \{positive\}, definite hermitian matrix. If $M=\left[M_{\alpha j}\right], N=\left[N_{\alpha j}\right],(\alpha=1, \cdots, n ; j=$ $1, \cdots, k)$; are $n \times k$ matrices, for typographical simplicity the symbol $(M ; N)$ is used to denote the $2 n \times k$ matrix whose $j$ th column has elements $M_{1 j}, \cdots M_{n j}, N_{1 j}, \cdots, N_{n j}$. If a matrix function $M(t)$ is a.c. (absolutely continuous) on an interval $[a, b]$, then $M^{\prime}(t)$ denotes the matrix of derivatives at values where these derivatives exist, and zero elsewhere. If $M(t)$ is (Lebesgue) integrable on $[a, b]$, then $\int_{a}^{b} M(t) d t$ signifies the matrix of integrals of respective elements of $M(t)$. For a given compact interval $[a, b]$ on the real line the symbols $\mathbb{B}_{m n}[a, b], \mathfrak{R}_{m n}^{\infty}[a, b]$, and $\mathbb{B}_{m n}^{2}[a, b]$ denote respectively the class of $m \times n$ matrix functions $M(t)=\left[M_{\alpha \beta}(t)\right]$ which on $[a, b]$ are (Lebesgue) inte- 
grable, (Lebesgue) measurable and essentially bounded, and measurable with $\left|M_{\alpha \beta}(t)\right|^{2}$ integrable. For $n=1$ the double subscript $m n$ is reduced to merely $m$. If matrix functions $M(t)$ and $N(t)$ are equal a.e. (almost everywhere) on a common interval of definition, we write simply $M(t)=N(t)$. An $m \times n$ matrix function $M(t)$ is said to be locally a.c. if $M$ is a.c. for arbitary compact subintervals [a,b].

2. Basic properties of differential systems. In the following, we shall consider a vector differential equation

$$
\Im y^{\prime}(t)+\mathfrak{U}(t) y(t)=0
$$

where $\mathfrak{\Im}$ and $\mathfrak{A}$ are $2 n \times 2 n$ matrix functions on the real line $\boldsymbol{R}=$ $(-\infty, \infty)$ of the form

$$
\mathfrak{\Im}=\left[\begin{array}{cc}
0 & -E_{n} \\
E_{n} & 0
\end{array}\right], \quad \mathfrak{A}(t)=\left[\begin{array}{ll}
C(t) & -D(t) \\
-A(t) & -B(t)
\end{array}\right],
$$

and the $n \times n$ matrix functions $A, B, C, D$ satisfy the following condition.

$\left(\mathfrak{S}_{1}\right) \quad A(t), B(t), C(t), D(t)$ are of class $\mathfrak{Q}_{n n}[\alpha, b]$ for arbitrary compact subintervals $[a, b]$ on $\boldsymbol{R}$.

For $u, v n$-dimensional vector functions and $y=(u ; v)$, equation (2.1) may be written as the vector system

$$
\begin{aligned}
-v^{\prime}(t)+C(t) u(t)-D(t) v(t) & =0, \\
u^{\prime}(t)-A(t) u(t)-B(t) v(t) & =0 .
\end{aligned}
$$

Throughout the paper it will be understood that "solution" is in the Carathéodory sense; that is, locally a.c. vector functions which satisfy a.e. the given equations. Corresponding to (2.1') we have the matrix differential system

$$
\begin{aligned}
-V^{\prime}(t)+C(t) U(t)-D(t) V(t) & =0, \\
U^{\prime}(t)-A(t) U(t)-B(t) V(t) & =0,
\end{aligned}
$$

where $U$ and $V$ are $n \times n$ matrix functions on $\boldsymbol{R}$.

Associated with this linear equation is the Riccati matrix differential equation

$$
W^{\prime}(t)+W(t) A(t)+D(t) W(t)+W(t) B(t) W(t)-C(t)=0,
$$

for which we have the well-known result (see, for example, [7, p. 101 , or $8, \mathrm{p} .11])$, that $W(t)$ is a solution of (2.3) on a nondegenerate subinterval $I$ of $\boldsymbol{R}$ if and only if there exists a solution $Y(t)=$ $(U(t) ; V(t))$ of $\left(2.1_{\mathrm{M}}\right)$ such that on this subinterval $U(t)$ is nonsingular 
and $W(t)=V(t) U^{-1}(t)$. Moreover, for $Y(t)$ and $W(t)$ thus related we have that $U(t)$ is a solution of the linear matrix differential equation

$$
U^{\prime}(t)=[A(t)+B(t) W(t)] U(t)
$$

which is nonsingular for each $t \in I$.

We shall be concerned with differential equations (2.1) and (2.3) for which the following hypothesis is satisfied.

$\left(\mathfrak{S}_{2}\right)$ The matrix functions $A(t), B(t), C(t), D(t)$ are periodic of period $\omega,(\omega>0)$, on $R$.

Now if hypotheses $\left(\mathfrak{F}_{1}\right)$ and $\left(\mathscr{F}_{2}\right)$ are satisfied, and there exists on $\boldsymbol{R}$ a solution $W(t)$ of (2.3) which is periodic of period $\omega$, then the coefficient matrix $A(t)+B(t) W(t)$ of (2.4) is periodic of period $\omega$. Consequently, if $Y(t)=(U(t) ; V(t))$ is a solution of $\left(2.1_{\mathrm{M}}\right)$ with $U(t)$ nonsingular and $W(t)=V(t) U^{-1}(t)$, then by the Floquet theory (see, for example [7, Sec. VII. 6]), there exists a nonsingular matrix function $P(t)$ of period $\omega$ and a constant matrix $S$ such that

$$
U(t)=P(t) \exp \{t S\}
$$

That is, $Y(t)=(U(t) ; V(t))=(U(t) ; W(t) U(t))$ is such that

$$
Y(t+\omega)=Y(t) K,
$$

where $K$ is the nonsingular matrix

$$
K=\exp \{\omega S\} \text {. }
$$

Conversely, if $Y(t)=(U(t) ; V(t))$ is a solution of $\left(2.1_{\mathrm{M}}\right)$ with $U(t)$ nonsingular, and (2.6) holds with $K$ a nonsingular matrix, then $W(t)=$ $V(t) U^{-1}(t)$ is periodic of period $\omega$. That is, we have the following result.

THEOREM 2.1. If hypotheses $\left(\mathfrak{S}_{1}\right),\left(\mathfrak{S}_{2}\right)$ are satisfied, then the Riccati matrix differential equation (2.3) has a solution $W(t)$ which is periodic with period $\omega$ if and only if there exists a solution $Y(t)=(U(t) ; V(t))$ of $\left(2.1_{\mathrm{M}}\right)$ with $U(t)$ nonsingular and which satisfies (2.6) with a nonsingular matrix $K$.

If the roles of $u$ and $v$ are interchanged in (2.1), then $\widetilde{y}=(\widetilde{u} ; \widetilde{v})=$ $(v ; u)$ is a solution of the differential system

$$
J \widetilde{y}^{\prime}+\tilde{\mathfrak{Y}}(t) \tilde{y}(t)=0,
$$

where

$$
\tilde{\mathscr{X}}(t)=\left[\begin{array}{rr}
\widetilde{C}(t) & -\widetilde{D}(t) \\
-\widetilde{A}(t) & -\widetilde{B}(t)
\end{array}\right]
$$


with $\widetilde{A}=-D, \widetilde{B}=C, \widetilde{C}=B, \widetilde{D}=-A$. In the self-adjoint case, wherein $D=A^{*}$, the equation (2.8) has been called the obverse to (2.1), (see [6, Sec. 2]). Clearly, if $W(t)$ is a solution of (2.3) that is nonsingular on a subinterval $I_{0}$, then $\tilde{W}(t)=W^{-1}(t)$ is a solution on $I_{0}$ of the Riccati matrix differential equation

$$
\widetilde{W}^{\prime}(t)+\widetilde{W}(t) \tilde{A}(t)+\widetilde{D}(t) \widetilde{W}(t)+\widetilde{W}(t) \widetilde{B}(t) \widetilde{W}(t)-\widetilde{C}(t)=0,
$$

associated with (2.8) as (2.3) is associated with (2.1).

Correspondingly, if $\hat{y}=(\hat{u} ; \hat{v})=(v ;-u)$, the equation (2.1) becomes

$$
J \hat{y}^{\prime}(t)+\hat{\mathfrak{A}}(t) \hat{y}(t)=0
$$

where

$$
\hat{\mathfrak{A}}(t)=\left[\begin{array}{rr}
\hat{C}(t) & -\hat{D}(t) \\
-\hat{A}(t) & -\hat{B}(t)
\end{array}\right],
$$

with $\hat{A}=-D, \hat{B}=-C, \hat{C}=-B, \hat{D}=-A$. Again, in the self-adjoint case, equation (2.11) has been termed reciprocal to (2.1), (see [2, Sec. 2]). Also, corresponding to the above comment, if $W(t)$ is a solution of (2.3) which is nonsingular on a subinterval $I_{0}$, then $\hat{W}(t)=$ $-W^{-1}(t)$ is a solution on $I_{0}$ of the Riccati matrix integral equation

$$
\hat{W}^{\prime}(t)+\hat{W}(t) \hat{A}(t)+\hat{D}(t) \hat{W}(t)+\hat{W}(t) \hat{B}(t) \hat{W}(t)-\hat{C}(t)=0 .
$$

3. Hamiltonian differential systems. Attention will now be directed to equations (2.1) whose coefficients satisfy the following hypothesis.

$\left(\mathfrak{S}_{3}\right) \quad B(t) \equiv B^{*}(t), C(t) \equiv C^{*}(t), D(t) \equiv A^{*}(t)$, and the matrix functions $A, B, C$ are of class $\mathfrak{R}_{n n}[a, b]$ on arbitrary compact subintervals $[a, b]$ of $R$.

Under hypothesis $\left(\mathfrak{S}_{3}\right)$ the matrix function $\mathfrak{A}(t)$ of $(2.2)$ is hermitian, and such equations are termed Hamiltonian vector differential equations. In particular, if the coefficients of (2.1) satisfy $\left(\mathfrak{S}_{3}\right)$ then this hypothesis is also satisfied by the coefficients of the obverse equation (2.8) and the reciprocal equation (2.11).

In particular, when $\left(\mathfrak{S}_{3}\right)$ is satisfied the vector system $\left(2.1^{\prime}\right)$ becomes

$$
\begin{aligned}
-v^{\prime}(t)+C(t) u(t)-A^{*}(t) v(t) & =0, \\
u^{\prime}(t)-A(t) u(t)-B(t) v(t) & =0 .
\end{aligned}
$$

Similarly, the system $\left(2.1_{M}\right)$ becomes

$$
\begin{aligned}
-V^{\prime}(t)+C(t) U(t)-A^{*}(t) V(t) & =0, \\
U^{\prime}(t)-A(t) U(t)-B(t) V(t) & =0,
\end{aligned}
$$


and the associated Riccati matrix equation (2.3) is

$$
W^{\prime}(t)+W(t) A(t)+A^{*}(t) W(t)+W(t) B(t) W(t)-C(t)=0 .
$$

If $y_{\alpha}=\left(u_{\alpha} ; v_{\alpha}\right)$ are solutions of $\left(3.1^{\prime}\right)$, then $y_{2}^{*}(t) \Im y_{1}(t)=v_{2}^{*}(t) u_{1}(t)-$ $u_{2}^{*}(t) v_{1}(t)$ is a constant on $\boldsymbol{R}$; if the value of this constant is zero, the solutions are said to be (mutually) conjoined or conjugate. Correspondingly, if $Y_{\alpha}(t)=\left(U_{\alpha}(t) ; V_{\alpha}(t)\right),(\alpha=1,2)$, are solutions of $\left(3.1_{M}\right)$, then the $n \times n$ matrix $Y_{2}^{*}(t) \mathfrak{\Im} Y_{1}(t)=V_{2}^{*}(t) U_{1}(t)-U_{2}^{*}(t) V_{1}(t)$ is constant. If the column vectors of $Y(t)=(U(t) ; V(t))$ are linearly independent solutions of $\left(3.1^{\prime}\right)$ and $Y^{*}(t) \Im Y(t) \equiv 0$, then $Y(t)$ is called a conjoined basis for $\left(3.1^{\prime}\right)$ or $\left(3.1_{\mathrm{M}}\right)$. From the form of (3.2) it is immediate that a solution $W(t)$ is hermitian throughout its interval of existence if and only if there exists a value $\tau$ on this interval such that $W(\tau)$ is hermitian. Moreover, for $(U ; V)$ a solution of $\left(3.1_{\mathrm{M}}\right)$ such that $W=V U^{-1}$ we have $W^{*}-W=U^{*-1}\left[V^{*} U-U^{*} V\right] U^{-1}$, and consequently $W$ is an hermitian solution of (3.2) if and only if an associated solution $Y(t)=(U(t) ; V(t))$ of $\left(3.1^{M}\right)$ satisfying $W=V U^{-1}$ is a conjoined basis for $\left(2.1^{\prime}\right)$.

In the following discussion we shall also assume the following hypotheses.

$\left(\mathfrak{S}_{4}\right) \quad B(t) \geqq 0$ for $t$ a.e. on $\boldsymbol{R}$.

( $\left.\mathfrak{S}_{5}\right)$ (3.1') is disconjugate on $\boldsymbol{R}$; that is, if $-\infty<a<b<\infty$ and $(u ; v)$ is a solution of (3.1') satisfying $u(a)=0=u(b)$, then $u(t)=0$ for $t \in[a, b]$.

( $\left.\mathfrak{S}_{6}\right)\left(3.1^{\prime}\right)$ is identically normal on $\boldsymbol{R}$; that is, if $(u(t) \equiv 0, v(t))$ is a solution of $\left(3.1^{\prime}\right)$ on a nondegenerate subinterval $[a, b]$ of $\boldsymbol{R}$, then also $v(t) \equiv 0$ on $[a, b]$ and $u \equiv 0, v \equiv 0$ on $\boldsymbol{R}$.

The following theorem presents known results on the existence of principal solutions of $\left(3.1^{\prime}\right)$ at $\pm \infty$, and corresponding distinguished solutions of (3.2), (see, for example, [7, Secs. VII: 3, VII: 5; in particular, Theorem VII: 3.4, VII: 5.4, and Problem VII: 5.8] or [8, Sec. IV. 8]).

THEOREM 3.1. Suppose that hypotheses $\left(\mathfrak{S}_{3}\right),\left(\mathfrak{S}_{4}\right),\left(\mathfrak{S}_{\mathfrak{F}_{5}}\right)$ and $\left(\mathfrak{S}_{6}\right)$ hold. If $c \in \boldsymbol{R}$ and $d<c<b$, then there exist unique solutions $Y(\mid c, b)=(U(\mid c, b) ; V(\mid c, b)), Y(\mid d, c)=(U(\mid d, c) ; V(\mid d, c))$ of $\left(3.1_{\mathrm{M}}\right)$ satisfying the boundary conditions

$$
U(c \mid c, b)=E, \quad U(b \mid c, b)=0 ; \quad U(c \mid d, c)=E, \quad U(d \mid d, c)=0 ;
$$

in particular, $Y(\mid c, b)$ and $Y(\mid d, c)$ are conjoined bases for $\left(3.1_{\mathrm{M}}\right)$. The matrix functions $U(t \mid c, b)$ and $U(t \mid d, c)$ are nonsingular on the respective subintervals $[c, b)$ and $(d, c]$, so that

$$
W(t \mid c, b)=V(t \mid c, b) U^{-1}(t \mid c, b)
$$


and $W(t \mid d, c)=V(t \mid d, c) U^{-1}(t \mid d, c)$ are hermitian solutions of (3.2) on these respective subintervals. Also, if $d<\hat{d}<c<\hat{b}<b$, then

$$
W(c \mid c, \hat{b}) \leqq W(c \mid c, b) \leqq W(c \mid d, c) \leqq W(c \mid \hat{d}, c),
$$

so that $\Gamma_{c, \infty}=\lim _{b \rightarrow \infty} W(c \mid c, b)$ and $\Gamma_{c,-\infty}=\lim _{d \rightarrow-\infty} W(c \mid d, c)$ exist and satisfy $\Gamma_{c, \infty} \leqq \Gamma_{c,-\infty}$. Moreover, if $W_{c, \infty}(t)$ and $W_{c,-\infty}(t)$ are the solutions of (3.2) satisfying $W_{c, \infty}(c)=\Gamma_{c, \infty}$ and $W_{c,-\infty}(c)=\Gamma_{c,-\infty}$, then $W_{c, \infty}(t)$ and $W_{c,-\infty}(t)$ are hermitian solutions of (3.2) on $\boldsymbol{R}$ which are independent of the value $c$, and consequently will be denoted by $W_{\infty}(t)$ and $W_{-\infty}(t)$ respectively. Finally, $W_{\infty}(t) \leqq W_{-\infty}(t)$ for $t \in \boldsymbol{R}$, and an hermitian solution $W(t)$ of (3.2) has interval of existence equal to $\boldsymbol{R}$ if and only if there exists a value $\tau$ such that $W_{\infty}(\tau) \leqq$ $W(\tau) \leqq W_{-\infty}(\tau)$, in which case $W_{\infty}(t) \leqq W(t) \leqq W_{-\infty}(t)$ throughout $\boldsymbol{R}$.

It is to be commented that in the cited references to Reid [7] the hypothesis corresponding to $\left(\mathfrak{S}_{3}\right)$ required the coefficient matrices to be of class $\mathfrak{R}_{n n}^{\infty}[a, b]$. There the hypothesis was thus formulated in order that the associated Dirichlet functional $J[\eta]$ might be considered in the Hilbert space setting wherein the canonical variable $\zeta$ satisfying with $\eta$ the differential equation $\eta^{\prime}-A(t) \eta-B(t) \zeta=0$ is of class $\mathfrak{R}_{n}^{2}[a, b]$. As far as solutions of the differential systems (3.1'), (3.1 $)$ and (3.2) are concerned, however, like results under the hypotheses of the present paper are derivable by identical proofs, where now a canonical variable $\zeta$ associated with an a.c. $\eta$ is assumed to be of class $\mathbb{R}_{n}^{\infty}[a, b]$.

The matrix functions $W_{\infty}$ and $W_{-\infty}$ are called the distinguished solutions of (3.2) at $\infty$ and $-\infty$, respectively; corresponding solutions $Y_{\infty}=\left(U_{\infty} ; V_{\infty}\right)$ and $Y_{-\infty}=\left(U_{-\infty} ; V_{-\infty}\right)$ of $\left(3.1_{\mathrm{M}}\right)$ satisfying $W_{\infty}=V_{\infty} U_{\infty}^{-1}$ and $W_{-\infty}=V_{-\infty} U_{-\infty}^{-1}$ are called principal solutions of $\left(3.1_{\mathrm{M}}\right)$ at the respective values $\infty$ and $-\infty$. For some historical comments on these concepts the reader is referred to [7; p. 398]. In particular, since

$$
W_{-\infty}-W_{\infty}=U_{-\infty}^{*-1}\left[V_{-\infty}^{*} U_{\infty}-U_{-\infty}^{*} V_{\infty}\right] U_{\infty}^{-1},
$$

it follows that either $W_{-\infty}(t)-W_{\infty}(t)>0$ for all $t \in R$ or for each $\tau$ the matrix $V_{-\infty}^{*}(\tau) U_{\infty}(\tau)-U_{-\infty}^{*}(\tau) V_{\infty}(\tau)$ is singular. In this latter case the conjoined bases determined by $Y_{\infty}(t)$ and $Y_{-\infty}(t)$ have at least one vector function in common. That is, there exist nonzero $n$-dimensional vectors $\xi_{\infty}$ and $\xi_{-\infty}$ such that $Y_{\infty}(t) \xi_{\infty} \equiv Y_{-\infty} \xi_{-\infty}$, in which case in geometric terminology the values $-\infty$ and $\infty$ are mutually conjugate with respect to $\left(3.1^{\prime}\right)$.

In case $W_{-\infty}(t)-W_{\infty}(t)<0$ for $t \in \boldsymbol{R}$, upon possibly replacing $Y_{\infty}(t)$ by $Y_{\infty}(t) K_{\infty}$ or $Y_{-\infty}(t)$ by $Y_{-\infty}(t) K_{-\infty}, K_{\infty}$ and $K_{-\infty}$ nonsingular $n \times n$ matrices, one may normalize these principal solutions so that 


$$
V_{-\infty}^{*}(t) U_{\infty}(t)-U_{-\infty}^{*}(t) V_{\infty}(t) \equiv-E \text {. }
$$

In this instance the $2 n \times 2 n$ matrix function

$$
\mathscr{Y}(t)=\left[\begin{array}{ll}
U_{-\infty}(t) & U_{\infty}(t) \\
V_{-\infty}(t) & V_{\infty}(t)
\end{array}\right]
$$

is such that $\mathscr{Y}^{*}(t) \mathfrak{Y}(t) \equiv \Im$; that is, for each $t \in R$ the matrix (3.5) is symplectic. In particular, we have that following result.

THEOREM 3.2. If hypotheses $\left(\mathfrak{S}_{3}\right),\left(\mathfrak{F}_{4}\right),\left(\mathfrak{S}_{5}\right),\left(\mathfrak{S}_{6}\right)$ are satisfied, and $W_{-\infty}(t)-W_{\infty}(t)>0$ for $t \in \boldsymbol{R}$, then $Y(t)$ defined by (3.5) is a fundamental matrix of solutions for $\left(3.1^{\prime}\right)$.

Now suppose that $\left(\mathscr{K}_{3}\right)$ is satisfied, with $B(t) \geqq 0$ and $C(t) \geqq 0$ for $t$ a.e. on $\boldsymbol{R}$, while both (3.1') and its obverse are identically normal. With the aid of an argument similar to that of $\S 8$ of [5], it may be established that the nonnegative matrix function $V_{\infty}^{*}(t) B(t) V_{\infty}(t)+$ $U_{\infty}^{*}(t) C(t) U_{\infty}(t)$ is integrable on $[c, \infty)$ for arbitrary $c \in \boldsymbol{R}$, and

$$
-U_{\infty}^{*}(c) W_{\infty}(c) U_{\infty}(c)=\int_{c}^{\infty}\left\{V_{\infty}^{*}(t) B(t) V_{\infty}(t)+U_{\infty}^{*}(t) C(t) U_{\infty}(t)\right\} d t .
$$

Moreover, in view of the assumption that (3.1') and its obverse are both identically normal, the right-hand member of (3.6) is a positive definite matrix for $c \in \boldsymbol{R}$, and hence $W_{\infty}(t)<0$ on $\boldsymbol{R}$. By an analogous argument it may be established that $W_{-\infty}(t)>0$, and, in particular, $W_{-\infty}(t)-W_{\infty}(t)>0$ for $t \in \boldsymbol{R}$. Also, for the distinguished solutions $\widetilde{W}_{\infty}(t)$ and $\widetilde{W}_{-\infty}(t)$ of the Riccati matrix differential equation associated with the obverse system we have $\widetilde{W}_{\infty}(t)=\left[W_{\infty}(t)\right]^{-1}<0$ and $\widetilde{W}_{-\infty}(t)=$ $\left[W_{-\infty}(t)\right]^{-1}>0$, so that also $\widetilde{W}_{-\infty}(t)-\widetilde{W}_{\infty}(t)>0$.

In case hypothesis $\left(\mathfrak{S}_{3}\right)$ is satisfied, while $B(t) \geqq 0$ and $C(t) \leqq 0$ for $t$ a.e. on $\boldsymbol{R}$, and both (3.1') and its reciprocal equation are identically normal, then results of Ahlbrandt $[1,2]$ may be used to establish relations between distinguished solutions of $\left(3.1_{M}\right)$ and the corresponding Riccati matrix differential equation for the obverse system.

4. Hamiltonian systems with periodic coefficients. We shall now consider Hamiltonian systems (3.1') under the following additional hypotheses.

$\left(\mathfrak{S}_{7}\right)$ The matrix functions $A(t), B(t), C(t)$ are periodic of period $\omega,(\omega>0)$, on $R$.

The following theorem presents a basic result for such systems.

THEOREM 4.1. If hypotheses $\left(\mathfrak{S}_{\mathfrak{Z}_{3}}\right),\left(\mathfrak{S}_{4}\right),\left(\mathfrak{S}_{5}\right),\left(\mathfrak{S}_{6}\right)$ and $\left(\mathfrak{S}_{7}\right)$ are 
satisfied, then the distinguished solutions $W_{\infty}(t)$ and $W_{-\infty}(t)$ of (3.2) are periodic, of period $\omega$.

This result is a ready consequence of the fact that the solutions $Y(\mid c, b)$ and $Y(\mid d, c)$ of $\left(3.1_{\mathrm{M}}\right)$ determined by the boundary conditions (3.3) are such that

$$
\begin{aligned}
& Y(t+\omega \mid c+\omega, b+\omega)=Y(t \mid c, b), \\
& Y(t-\omega \mid d-\omega, c-\omega)=Y(t \mid d, c)
\end{aligned}
$$

so that from the results of Theorem 3.1 it follows that

$$
W(t+\omega \mid c+\omega, b+\omega)=W(t \mid c, b)
$$

and $W(t-\omega \mid d-\omega, c-\omega)=W(t \mid d, c)$, together with the fact that $W_{c, \infty}(t)$ and $W_{c,-\infty}(t)$ are independent of the value $c$. In view of the domination of any hermitian solution of (3.2) by the distinguished solutions $W_{\infty}(t)$ and $W_{-\infty}(t)$, we have the following particular result.

CoRollary. Under the hypotheses of Theorem 4.1, if $W(t)$ is any hermitian solution of (3.2) which is periodic, of any period, then $W_{\infty}(t) \leqq W(t) \leqq W_{-\infty}(t)$ for $t \in \boldsymbol{R}$.

Consequently, whenever the hypotheses of Theorem 4.1 are satisfied it follows from the discussion of $\S 2$ preceding Theorem 2.1 that there exist nonsingular matrices $P_{\infty}(t), P_{-\infty}(t)$ of period $\omega$, and constant matrices $S_{\infty}$ and $S_{-\infty}$ such that

$$
U_{\infty}(t)=P_{\infty}(t) \exp \left[t S_{\infty}\right\}, \quad U_{-\infty}(t)=P_{-\infty}(t) \exp \left\{t S_{-\infty}\right\} .
$$

For any such representations an eigenvalue of $S_{\infty}$ or $S_{-\infty}$ is called a characteristic exponent for the corresponding matrix equation $U^{\prime}(t)=$ $\left[A(t)+B(t) W_{\infty}(t)\right] U(t) \quad$ or $\quad U^{\prime}(t)=\left[A(t)+B(t) W_{-\infty}(t)\right] U(t)$. In particular, the characteristic exponents are not determined uniquely, but are specified to within integral multiples of $2 \pi i / \omega$.

THEOREM 4.2. Suppose that hypotheses $\left(\mathfrak{S}_{3}\right),\left(\mathfrak{S}_{4}\right),\left(\mathfrak{S}_{5}\right),\left(\mathfrak{S}_{6}\right)$ and $\left(\mathscr{S}_{7}\right)$ are satisfied and $W_{-\infty}(t)-W_{\infty}(t)>0$ for $t \in \boldsymbol{R}$. If the corresponding principal solutions $\left(U_{\infty} ; V_{\infty}\right)$ and $\left(U_{-\infty} ; V_{-\infty}\right)$ of $\left(3.1_{\mathrm{M}}\right)$ are normalized to satisfy (3.4), and

$$
U_{\infty}(t)=P_{\infty}(t) \exp \left\{t S_{\infty}\right\}, U_{-\infty}(t)=P_{-\infty}(t) \exp \left\{t S_{-\infty}\right\}
$$

are representations (4.1) for $U_{\infty}(t)$ and $U_{-\infty}(t)$, respectively, then the fundamental matrix solution $\mathscr{Y}(t)$ of $\left(3.1_{\mathrm{M}}\right)$ given by (3.5) has Floquet representation 


$$
\mathscr{Y}(t)=\mathscr{P}(t) \exp \{t \mathscr{S}\}
$$

where

$$
\mathscr{P}(t)=\left[\begin{array}{ll}
P_{-\infty}(t) & P_{\infty}(t) \\
W_{-\infty}(t) P_{-\infty}(t) & W_{\infty}(t) P_{\infty}(t)
\end{array}\right], \quad \mathscr{S}=\left[\begin{array}{ll}
S_{-\infty} & 0 \\
0 & S_{\infty}
\end{array}\right] .
$$

Moreover, $\exp \left\{t S_{-\infty}\right\} \exp \left\{t S_{\infty}^{*}\right\}=E$, so that if $\mu_{1}^{\infty}, \cdots, \mu_{n}^{\infty}$ and $\mu_{1}^{-\infty}, \cdots$, $\mu_{n}^{-\infty}$ are characteristic exponents of $U_{\infty}(t)$ and $U_{-\infty}(t)$, respectively, then they may be so ordered that $\mu_{\alpha}^{-\infty}+\mu_{\alpha}^{\infty}$ is an integral multiple of $2 \pi i / \omega$ for $\alpha=1, \cdots, n$.

The representation (4.3) is an immediate consequence of the fact that

$$
\mathscr{Y}(t)=\left[\begin{array}{ll}
E & E \\
W_{-\infty}(t) & W_{\infty}(t)
\end{array}\right]\left[\begin{array}{ll}
U_{-\infty}(t) & 0 \\
0 & U_{\infty}(t)
\end{array}\right],
$$

and the representations (4.1) for $U_{\infty}(t)$ and $U_{-\infty}(t)$. Moreover, if $\left(U_{\infty} ; V_{\infty}\right)$ and $\left(U_{-\infty} ; V_{-\infty}\right)$ are normalized to satisfy (3.4) then

$$
U_{-\infty}^{*}(t)\left[W_{-\infty}(t)-W_{\infty}(t)\right] U_{\infty}(t) \equiv E,
$$

so that also $\left[W_{-\infty}(t)-W_{\infty}(t)\right] U_{\infty}(t) U_{-\infty}^{*}(t) \equiv E$. Consequently, if $M_{\infty}=$ $\exp \left\{\omega S_{\infty}\right\}$ and $M_{-\infty}=\exp \left\{\omega S_{-\infty}\right\}$, then also

$$
\begin{aligned}
E & \equiv\left[W_{-\infty}(t+\omega)-W_{\infty}(t+\omega)\right] U_{\infty}(t+\omega) U_{-\infty}^{*}(t+\omega), \\
& \equiv\left[W_{-\infty}(t)-W_{\infty}(t)\right] U_{\infty}(t) M_{\infty} M_{-\infty}^{*} U_{-\infty}^{*}(t),
\end{aligned}
$$

and as $W_{-\infty}(t)-W_{\infty}(t)$ is nonsingular it follows that $U_{\infty}(t) U_{-\infty}^{*}(t) \equiv$ $U_{\infty}(t) M_{\infty} M_{-\infty}^{*} U_{-\infty}^{*}(t)$. Consequently, $M_{\infty} M_{-\infty}^{*}=E$, so that $\lambda$ is an eigenvalue of $M_{\infty}$ if and only if $1 / \lambda$ is an eigenvalue of $M_{-\infty}^{*}$ and $1 / \bar{\lambda}$ is an eigenvalue of $M_{-\infty}$

5. Comments. As mentioned in the Introduction, the goal of this paper is quite distinct from that of Bucy [4]. It is also to be noted that the hypotheses of the two papers are quite distinct. For the present discussion the assumptions of definiteness and some type of normality are basic, whereas these aspects apparently do not enter into Bucy's treatment. On the other hand, Bucy requires that the assumed periodic solutions $P_{+}$and $P_{-}$of his Riccati matrix differential equation are invertible, while the present discussion does not involve any assumption of nonsingularity of the distinguished solutions $W_{\infty}$ and $W_{-\infty}$. Also, Bucy restricts attention to real Hamiltonian systems, while in the present discussion one may treat equally well the case of systems with complex coefficients. Finally, in [4] it is assumed that the characteristic roots of the matrix of the associated 
constant Hamiltonian system are simple with real parts not equal to zero, and no such condition is involved in the present discussion.

In regard to the hypotheses $\left(\mathscr{S}_{5}\right)$ and $\left(\mathscr{S}_{6}\right)$ of the present paper, the following remarks are pertinent. In general for Hamiltonian systems satisfying hypotheses $\left(\mathfrak{S}_{4}\right)$ and $\left(\mathfrak{S}_{6}\right)$, a necessary and sufficient condition for the existence of $W_{\infty}$ or $W_{-\infty}$ is the disconjugacy of (3.1') in a neighborhood of the respective value $\infty$ or $-\infty$, (see, for example, [7, Sec. VII: 3]), and for such systems which are periodic either of these conditions is equivalent to disconjugacy on the entire interval $(-\infty, \infty)$. This is, a necessary condition that either $W_{\infty}$ or $W_{-\infty}$ exists is that $\left(3.1^{\prime}\right)$ be disconjugate on $(-\infty, \infty)$, and this condition is sufficient for the existence of both of these distinguished solutions, and for each of them its maximal interval of existence is $(-\infty, \infty)$. As to condition $\left(\mathfrak{S}_{6}\right)$, it is stronger than the conditions of complete observability and complete controllability of Bucy, (see, for example [3, Chapter III]). In regard to these latter conditions, however, it is to be noted that in general for Hamiltonian systems satisfying hypothesis $\left(\mathfrak{F}_{4}\right)$ complete observability is the condition that for $t \in$ $(-\infty, \infty)$ there exists a value $a(t)<t$ such that the system is normal on $[a(t), t]$, while complete controllability is the condition that for $t \in(-\infty, \infty)$ there exists a value $b(t)>t$ such that the system is normal on $[t, b(t)]$, (see, [6; Sec. 7], and [8; Sec. V. 5]). Moreover, in the case of periodic Hamiltonian systems these conditions are readily seen to be equivalent to the existence of a single $t_{0}$ for which there exists a corresponding $a\left(t_{0}\right)<t_{0}$ or $b\left(t_{0}\right)>t_{0}$ such that the system is normal on the respective interval $\left[a\left(t_{0}\right), t_{0}\right]$ or $\left[t_{0}, b\left(t_{0}\right)\right]$, and consequently for such periodic systems there is automatically a condition of uniformity for these concepts.

It is to be noted also that in the above discussion the condition $\left(\mathfrak{S}_{6}\right)$ may be weakened to require merely that there exists a nonnegative integer $d$ such that on arbitrary non-degenerate subintervals $I$ of $R$ the order of abnormality of $\left(3.1^{\prime}\right)$ on $I$ is equal to $d$, and that the associated "truncated preferred reduced system of $\left(3.1^{\prime}\right)$ " is periodic. In this connection, the reader is referred to [6; Sec. 6, Theorem 6.3 in particular], and [8; Sec. IV: 8, Theorem IV: 8.5 in particular].

\section{REFERENCES}

1. C. D. Ahlbrandt, Equivalent boundary value problems for self-adjoint differential systems, J. Differential Equations, 9 (1971), 420-435.

2. - Principal and antiprincipal solutions of self-adjoint differential systems and their reciprocals, Rocky Mountain J. Math., 2 (1972), 169-182.

3. R. S. Bucy, and P. D. Joseph, Filtering for Stochastic Processes with Applications to Guidance, Wiley (Interscience), New York, 1968. 
4. R. S. Bucy, The problem of Hill for systems, Proceedings of the International Conference on Differential Equations, University of Southern California, Los Angeles, California, September 3-7, 1974, 65-94.

5. W. T. Reid, Principal solutions of non-oscillatory self-adjoint linear differential systems, Pacific J. Math., 8 (1958), 147-169.

6. Monotoneity properties of solutions of hermitian Riccati matrix differential equations, SIAM J. Math. Analysis, 1 (1970), 195-213.

7. — Ordinary Differential Equations, John Wiley and Sons, New York, 1971.

8. — Riccati Differential Equations, Academic Press, New York, 1972.

Received October 30, 1974 and in revised form January 17, 1975. The author was in no way responsible for the unfortunate delay in the publication of this paper.

UNIVERSITY OF OKLAHOMA

Present Address: Department of Mathematics, RLM 8-100

The University of Texas at Austin

Austin, Texas 78712 



\section{PACIFIC JOURNAL OF MATHEMATICS}

\section{EDITORS}

RICHARD ARENS (Managing Editor)

University of California

Los Angeles, CA 90024

R. A. BEAUMONT

University of Washington

Seattle, WA 98105

C. C. Moore

University of California

Berkeley, CA 94720

\section{J. DugundJI}

Department of Mathematics

University of Southern California

Los Angeles, CA 90007

R. Finn and J. Milgram

Stanford University

Stanford, CA 94305

\section{ASSOCIATE EDITORS}

\section{E. F. BECKENBACH}

B. H. NEUMANN

F. WoLF

K. YOSHIDA

\section{SUPPORTING INSTITUTIONS}

\author{
UNIVERSITY OF BRITISH COLUMBIA \\ CALIFORNIA INSTITUTE OF TECHNOLOGY \\ UNIVERSITY OF CALIFORNIA \\ MONTANA STATE UNIVERSITY \\ UNIVERSITY OF NEVADA \\ NEW MEXICO STATE UNIVERSITY \\ OREGON STATE UNIVERSITY \\ UNIVERSITY OF OREGON \\ OSAKA UNIVERSITY
}

\author{
UNIVERSITY OF SOUTHERN CALIFORNIA \\ STANFORD UNIVERSITY \\ UNIVERSITY OF HAWAII \\ UNIVERSITY OF TOKYO \\ UNIVERSITY OF UTAH \\ WASHINGTON STATE UNIVERSITY \\ UNIVERSITY OF WASHINGTON \\ AMERICAN MATHEMATICAL SOCIETY
}

The Supporting Institutions listed above contribute to the cost of publication of this Journal, but they are not owners or publishers and have no responsibility for its content or policies.

Mathematical papers intended for publication in the Pacific Jaurnal of Mathematics should be in typed form or offset-reproduced, (not dittoed), double spaced with large margins. Please do not use built up fractions in the text of your manuscript. You may however, use them in the displayed equations. Underline Greek letters in red, German in green, and script in blue. The first paragraph or two must be capable of being used separately as a synopsis of the entire paper. Items of the bibliography should not be cited there unless absolutely necessary, in which case they must be identified by author and Journal, rather than by item number. Manuscripts, in triplicate, may be sent to any one of the editors. Please classify according to the scheme of Math. Reviews, Index to Vol. 39. All other communications should be addressed to the managing editor, or Elaine Barth, University of California, Los Angeles, California, 90024.

The Pacific Journal of Mathematics expects the author's institution to pay page charges, and reserves the right to delay publication for nonpayment of charges in case of financial emergency

100 reprints are provided free for each article, only if page charges have been substantially paid. Additional copies may be obtained at cost in multiples of 50 .

The Pacific Journal of Mathematics is issued monthly as of January 1966. Regular subscription rate: $\$ 7200$ a year (6 Vols., 12 issues). Special rate: $\$ 36.00$ a year to individual members of supporting institutions.

Subscriptions, orders for back numbers, and changes of address should be sent to Pacific Journal of Mathematics, 103 Highland Boulevard, Berkeley, California, 94708.

PUBLISHED BY PACIFIC JOURNAL OF MATHEMATICS, A NON-PROFIT CORPORATION

Printed at Kokusai Bunken Insatsusha (International Academic Printing Co., Ltd.). 8-8, 3-chome, Takadanobaba, Shinjuku-ku, Tokyo 160, Japan.

Copyrit (C) 1975 by Pacific Journal of Mathematics Manufactured and first issued in Japan 


\section{Pacific Journal of Mathematics \\ Vol. 69, No. $2 \quad$ June, 1977}

Carol Alf and Thomas Alfonso O'Connor, Unimodality of the Lévy spectral

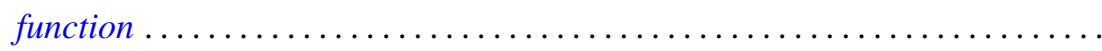

S. J. Bernau and Howard E. Lacey, Bicontractive projections and reordering of

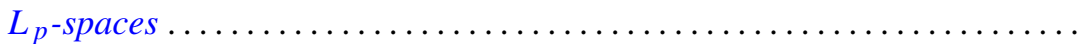

Andrew J. Berner, Products of compact spaces with bi-k and related spaces..... 303

Stephen Richard Bernfeld, The extendability and uniqueness of solutions of ordinary differential equations ...............................

Marilyn Breen, Decompositions for nonclosed planar m-convex sets ..........

Robert F. Brown, Cohomology of homomorphisms of Lie algebras and Lie

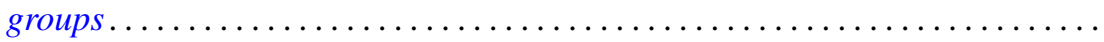

Jack Douglas Bryant and Thomas Francis McCabe, A note on Edelstein's iterative test and spaces of continuous functions ....................

Victor P. Camillo, Modules whose quotients have finite Goldie dimension ....... 333

David Downing and William A. Kirk, A generalization of Caristi's theorem with

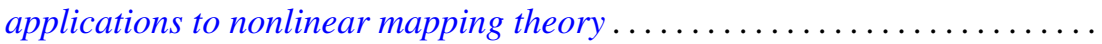

Daniel Reuven Farkas and Robert L. Snider, Noetherian fixed rings ...........

Alessandro Figà-Talamanca, Positive definite functions which vanish at

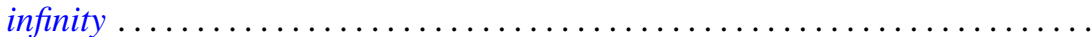

Josip Globevnik, The range of analytic extensions .................. 365

André Goldman, Mesures cylindriques, mesures vectorielles et questions de

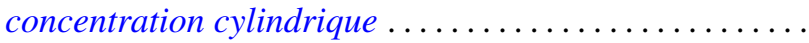

Richard Grassl, Multisectioned partitions of integers..........

Haruo Kitahara and Shinsuke Yorozu, A formula for the normal part of the

Laplace-Beltrami operator on the foliated manifold .... .

Marvin J. Kohn, Summability $R_{r}$ for double series .........

Charles Philip Lanski, Lie ideals and derivations in rings with involution ..

Solomon Leader, A topological characterization of Banach contractions . .

Daniel Francis Xavier O’Reilly, Cobordism classes of fiber bundles . .

James William Pendergrass, The Schur subgroup of the Brauer group . .

Howard Lewis Penn, Inner-outer factorization of functions whose Fourier series

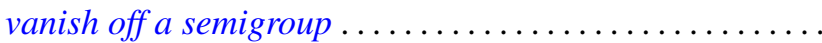

501

William T. Reid, Some results on the Floquet theory for disconjugate definite

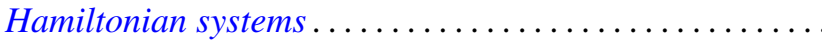

Caroll Vernon Riecke, Complementation in the lattice of convergence

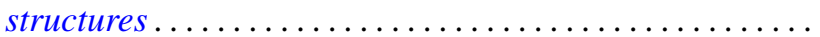

Louis Halle Rowen, Classes of rings torsion-free over their centers ......... 527

Manda Butchi Suryanarayana, A Sobolev space and a Darboux problem ....... 535

Charles Thomas Tucker, II, Riesz homomorphisms and positive linear maps.... 551

William W. Williams, Semigroups with identity on Peano continua ........... 557

Yukinobu Yajima, On spaces which have a closure-preserving cover by finite 